\title{
LA INDUSTRIA AEROESPACIAL: \\ UN ANÁLISIS DE SU PRODUCTIVIDAD EN MÉXICO
}

THE AEROSPACE INDUSTRY:

A PRODUCTIVITY ANALYSIS IN MEXICO

\author{
Karla Jeanine Melo Olguín*, Danaé Duana Ávila**, \\ Karina Valencia Sandoval ${ }^{\star \star \star}$
}

\begin{abstract}
*Estudiante de la Licenciatura en Comercio Exterior. Universidad Autónoma del Estado de Hidalgo. Área de Administración- Campus ICEA-La Concepción. Circuito la Concepción, km 2.5, San Juan Tilcuautla Municipio de San Agustín Tlaxiaca, Hidalgo, C.P. 42161, Tel: 01 (771) 717 2000, ext 4171. Email: duananos@yahoo.com.mx

** Doctor. Profesor-Investigador del Instituto de Ciencias Económico Administrativas. Universidad Autónoma del Estado de Hidalgo. Área de Administración- Campus ICEA-La Concepción. Circuito la Concepción, km 2.5, San Juan Tilcuautla Municipio de San Agustín Tlaxiaca, Hidalgo, C.P. 42161, Tel: 01 (771) 717 2000, ext 4171.

*** Doctora. Profesor-Investigador del Instituto de Ciencias Económico Administrativas. Universidad Autónoma del Estado de Hidalgo. Área de Administración- Campus ICEA-La Concepción. Circuito la Concepción, km 2.5, San Juan Tilcuautla Municipio de San Agustín Tlaxiaca, Hidalgo, C.P. 42161, Tel: 01 (771) 717 2000, ext 4171. Email: duananos@yahoo.com.mx, duana@uaeh.edu.mx.
\end{abstract}

Dirección para recibir correspondencia: duananos@yahoo.com.mx, duana@uaeh.edu.mx

Fecha de recibido: 13 de noviembre de 2017

Fecha de aceptación: 16 de enero de 2018 


\section{LA INDUSTRIA AEROESPACIAL: UNA ANÁLISIS DE SU PRODUCTIVIDAD EN MÉXICO}

\section{RESUMEN}

México necesita de nuevas estrategias que ayuden a su crecimiento económico, y con el paso de los años han surgido nuevas oportunidades que pueden aprovecharse para lograrlo. A pesar de poseer riqueza en amplios sectores, la sociedad está demandando otro tipo de productos que comúnmente se ofrecen.

El surgimiento de nuevas industrias ha elevado las expectativas que se tienen de nuestro país, por ejemplo la industria aeroespacial, que surgió no hace mucho tiempo y ahora es uno de las más productivas a nivel mundial. Aunado a ello, México tiene grandes ventajas frente a los mercados globales, ya que se ha caracterizado por la calidad en sus productos y servicios, compite con grandes potencias con Estados Unidos y Canadá, y recibe grandes inversiones que han elevado aún más su participación. Es por ello, que en la actualidad dicha industria está acaparando los mercados, ya que la demanda ha ido creciendo y en México se tienen las herramientas necesarias para realizar exportaciones y para incrementar el número de empresas de este giro alrededor del país.

PALABRAS CLAVE: Industria. Sectores económicos. Manufactura. Producto Interno Bruto.

\section{ABSTRACT}

Mexico needs new strategies to help its economic growth. Over the past years, new opportunities have arisen which can be used in order to achieve it. Despite having wealth in several sectors, society demands another type of products which that commonly offered.

The emerging new industries have created expectations in and about our country; for instance, the aerospace industry, which originated not long ago and it has become one of the most productive worldwide. Moreover, Mexico has great advantages compared to those global markets, because its products and services are distinctive for their quality; it also competes with power countries such as United States and Canada, It also receives investments which have cooperated to elevate its position. Therefore, such an industry is taking over markets, demand continue to increase and Mexico has the necessary tools for exporting and increasing the number of companies in this area all over the nation.

KEYWORDS: Industry. Economic sectors. Manufacturing. Gross Domestic Product. MELO-OLGUÍN K. J., DUANA-ÁVILA D., VALENCIA-SANDOVAL K. 


\section{INTRODUCCIÓN}

En México, la industria aeroespacial mexicana surgió hace 40 años y durante este tiempo ha buscado competir con las grandes potencias: Estados Unidos de América y Canadá. Sin embargo, se tiene obstáculos que no le permiten establecerse y además de sufrir altibajos que no le proporcionan estabilidad.

La industria aeroespacial es de las más nuevas y completas que están presentes en el mercado, los primeros datos acercan a Alberto Braniff en 1910 quien pagó alrededor de 25 mil pesos por la construcción de su avión que fue traído a México y se convirtió en el primer piloto mexicano en volar. Después de algunos años, en 1917 el piloto Horacio Ruiz Gaviño voló por primera vez una aeronave completamente fabricada en México: un biplano tipo Serie A con un motor Aztátl y una hélice Anáhuac y en aquel tiempo se construyeron 58 aeronaves en los Talleres Nacionales de Construcciones Aeronáuticas. Posteriormente en 1994 fue creada la compañía Mexicana de Aviación en Tampico, por George L. Rhil que más tarde se asoció con la Compañía Mexicana de Transportación Aérea para crear Mexicana de Aviación, logrando ser la cuarta aerolínea más antigua del mundo en servicio.

México realiza innumerables exportaciones a varios países, principalmente a Canadá y Estados Unidos, logrando ser el primer exportador de electrodomésticos de América Latina y el quinto lugar a nivel internacional, además de ocupar el primer puesto en exportaciones de autopartes; tan solo en 2015 las exportaciones sumaron un total de \$390 000000.00 (US\$), de acuerdo a fuentes de ProMéxico (2015).

Desde el año 2004, las cosas han ido cambiando para la industria aeroespacial, en aquel entonces había 65 plantas dedicadas a éste giro y con un total de 12500 empleados, mientras que para el año 2011 las cifras aumentaron a 260 plantas con 33000 trabajadores, es decir, 264\% más en la generación de empleos siendo la mejor evidencia del progreso que ha tenido dicha industria. Baja California es el estado que cuenta con el mayor número de plantas en México, con 42 y un total de 12,204 empleados. Todo esto es gracias a su ubicación, geográfica y a la cercanía que tiene con Estados Unidos. De la misma manera, Sonora cuenta con varias plantas: 21, y en los últimos puestos se encuentra Aguascalientes, Puebla y Yucatán. Las industrias del país están establecidas en distintas regiones, siendo favorable para el desarrollo de la misma en todo el país. Gran parte del crecimiento de la industria aeroespacial en México tiene origen en el Estado de Querétaro, debido a que en el 2005 se instaló la primer empresa MELO-OLGUÍN K. J., DUANA-ÁVILA D., VALENCIA-SANDOVAL K. 


\section{LA INDUSTRIA AEROESPACIAL: UNA ANÁLISIS DE SU PRODUCTIVIDAD EN MÉXICO}

aeroespacial "Bombardier", tomando tan solo 2 años para realizar su primer envío hacia Canadá. Se creó un proyecto que buscaba preparar a los primeros técnicos e ingenieros en dicho estado, para que pudieran producir las primeras aeropartes y en un futuro grandes aviones, dicha idea se extendió hasta el año 2012.

El principal problema radica en el poco valor que se le asigna a éste sector, actualmente el gobierno le está dando prioridad a empresas o industrias que aseguren su "estabilidad económica", como la automotriz, textil o agroalimentaria. Otra dificultad que enfrentan estas empresas reside en que el personal que ahí labora, en su mayoría no cuentan con licencia y su trabajo no siempre es valorado. Aunado a lo anterior, debe contemplarse que muchas empresas, incluso mexicanas prefieren hacer su inversión en industrias extranjeras, y es que consideran varios factores para hacerlo: desde tratados o acuerdos, popularidad, y lo que se pretende lograr es que México obtenga más mercado en diversos países y no sólo entre Estados de la República. El claro ejemplo es Querétaro, el objetivo no es que produzca y venda dentro del país, sino que vaya más allá, que cruce fronteras y le dé una nueva oportunidad a éste sector.

Pese a lo anterior, México cuenta con muchas herramientas que le permiten sobresalir dentro del concierto mundial, una de ellas es la mano de obra calificada derivada de la oferta educativa nacional, por ejemplo el Instituto Politécnico Nacional (IPN), la Universidad Autónoma de Nuevo León (UANL), y la Universidad Aeronáutica en Querétaro, creada en septiembre de 2007. Como es de esperarse, egresan muchos estudiantes preparados, que cuentan con los conocimientos y habilidades para desempeñarse en el campo laboral.

Sin embargo, varias décadas atrás (1970), en nuestro país se instalaron tres industrias importantes: maquiladora, automotriz y la aeroespacial. Esto permitió un amplio conocimiento en distintas disciplinas, propicio nuevas oportunidades de trabajo, pero que requieren conocimientos avanzados, asimismo otorgó nuevas puertas a empresarios, además benefició al sector educativo, ya que como se mencionó anteriormente hubo la apertura de licenciaturas con estrecha relación a este campo.

\section{La industria aeroespacial en Norteamérica}

La industria es una actividad económica que conforma el sector secundario y tiene por objetivo transformar la materia prima en productos o mercancías más sofisticadas. Las industrias 


\section{LA INDUSTRIA AEROESPACIAL: UNA ANÁLISIS DE SU PRODUCTIVIDAD EN MÉXICO}

actualmente se clasifican en dos: la industria extractiva y la de transformación, las cuales a su vez se subdividen de acuerdo a los productos que elaboran, en la primera se encuentra la minería y el petróleo, y por el contrario en la de transformación se localizan 47 tipos, entre las que destacan: la industria textil, la manufacturera, la automotriz, la tequilera y, en la actualidad, la aeroespacial.

La revolución industrial marcó el inicio de la gran transformación en el mundo, a partir del siglo XIX y durante el siglo XX e inició en Inglaterra con la utilización de máquinas y la aparición de la electricidad y el petróleo. Además, hubo un crecimiento de las empresas, ya no eran pequeñas, los bancos ejercían sobre las mismas, por lo que a esta revolución también se le llamó la "Era del Capitalismo Financiero"; sin embargo, durante este tiempo la industria aeroespacial no era tan relevante como ahora (FEMIA, 2013).

La industria aeroespacial es aquella que se dedica al diseño, fabricación, comercialización y mantenimiento de aviones. Al principio esta actividad predominaba en Estados Unidos y hasta ahora es el mayor consumidor de tecnología y productos aeroespaciales. Cuenta como empresas como Boeing, United Technologies Corporation y Lockheed Martin. Pero ésta industria ahora representa gran dinamismo y desarrollo para los países que la promueven, ya que su mercado representa alrededor de 450 mil millones de dólares a nivel mundial.

A partir de 1970, iniciaron las primeras empresas aeroespaciales en México; sin embargo, no tuvieron éxito al enfrentarse a grandes potencias como Estados Unidos y Canadá, por lo que no representaron gran avance. Fue hasta el 2004, cuando iniciaron nuevamente los trabajos, y durante este tiempo las grandes empresas habían trabajado en conjunto con el gobierno para lograr el auge que México requiere.

Asimismo, Canadá cuenta con la tecnología más desarrollada y está ubicada en el cuarto lugar en la industria aeronáutica. En 2005, alcanzó la cifra de ventas de 18,000 millones de dólares canadienses aproximadamente, empleando alrededor de 80,000 personas; su principal empresa, Bombardier, produce aviones que abastecen el 40\% del mercado. Este país es líder mundial en la producción de simuladores de vuelo y sistemas de entrenamiento, además de contar con el respaldo de la NASA desde hace tres décadas, aunado a esto, la industria aeroespacial canadiense tiene relación con la Agencia Espacial Europea desde hace dos décadas y cooperación bilateral con otros países europeos y asiáticos. 


\section{LA INDUSTRIA AEROESPACIAL: UNA ANÁLISIS DE SU PRODUCTIVIDAD EN MÉXICO}

Por el contrario, Europa cuenta con cuatro países con una excelente producción, tal es el caso de Alemania, Francia, Reino Unido e Italia, por lo que éste sector supera los 30 mil millones de euros y emplea a más de 430, 000 personas (FEMIA, 2015).

En el año 2009, las importaciones a nivel mundial fueron de 93,000 millones de dólares, siendo Alemania (15 mil millones de dólares en 2009), Estados Unidos y China (9 mil millones de dólares), e Irlanda (5 mil millones de dólares) los principales países importadores, donde México no figuraba entre los primeros 16 lugares. Sin embargo, las exportaciones de México en 2015 a Estados Unidos sumaron un total de 2,413 millones de dólares, ubicándose como el séptimo proveedor de ese país (FEMIA, 2015).

\section{Industria aeroespacial en México}

En México, la industria aeroespacial está logrando un buen posicionamiento a nivel global, siendo la Federación Mexicana de la Industria Aeroespacial A.C. (FEMIA), la encargada de agrupar a la mayor parte de las industrias aeroespaciales de toda la República Mexicana. El número de empresas aeroespaciales localizadas en México ha crecido, en el 2005 apenas había 61 empresas en el país, mientras que en el 2010 ya eran 238. Actualmente, la industria aeroespacial nacional integra a 260 empresas (FEMIA, 2010).

La Federación Mexicana de la Industria Aeroespacial busca promover a sus miembros en los diferentes mercados para elevar su competitividad, cuenta con alrededor de 58 asociados y 24 afiliados. Para contar con el reconocimiento que hasta ahora ha logrado, FEMIA ha tenido que pasar por diversas certificaciones que evalúen su buen funcionamiento, como la ISO14000 que se refiere a la Gestión Ambiental, lo cual muestra que efectivamente cumplen con las normas que protegen al medio ambiente, y las empresas que forman parte de ella están comprometidas a un buen uso y aplicación de sus productos.

Gracias a la creación de la Universidad de Aeronáutica en Querétaro y de la apertura de licenciaturas dirigidas a este rubro, la cantidad de profesionistas ha incrementado, tan solo en 2012 se graduaron 111.4 mil estudiantes de ingeniería y tecnología (Visión industrial, 2012).

Las exportaciones de partes de aeronaves ha incrementado desde el año 2010 a la actualidad, ya que en 2010 las exportaciones sumaron un total de 3266 millones de dólares y hasta el año 2013 la cantidad fue de 5,463 millones de dólares (FEMIA, 2010). 


\section{LA INDUSTRIA AEROESPACIAL: UNA ANÁLISIS DE SU PRODUCTIVIDAD EN MÉXICO}

La industria aeroespacial mexicana se enfoca a cinco áreas de trabajo, las cuales son las siguientes: diseño e ingeniería, manufactura de piezas y partes, mantenimiento de aviones, ensamble y reciclado y reconstrucción. Provee principalmente a Estados Unidos (81\%), en segundo lugar se ubican Francia y Alemania con el 2.8\%, respectivamente; y en tercer término, Canadá con $2.6 \%$ y Reino Unido también con el $2.6 \%$ del total de su producción. México está clasificado como el noveno proveedor para el mercado aeroespacial de Estados Unidos y el sexto para la Unión Europea. Actualmente, existen alrededor de 300 empresas del sector aeroespacial, de las cuales el $80 \%$ son manufactureras y el otro $20 \%$ ofrece servicios de diseño e ingeniería, así como de mantenimiento, reparación y operaciones.

\section{Tamaño de las empresas aeroespaciales en México}

El grueso de las empresas (43\%) son de tamaño mediano, pues emplean entre 51 y 250 trabajadores; el 29\% son pequeñas o micro empresas, las cuales contratan a menos de 50 empleados, y el $28 \%$ son grandes, siendo que en el país hay 15 empresas que emplean a más de 500 personas (Secretaría de Economía, 2015).

El establecimiento de la industria enfocada a las aeronaves, tiene presencia en 5 estados de la República, todos ellos con ventajas que facilitan la producción y el desarrollo de grandes empresas. Las principales regiones son: Baja California, Chihuahua, Sonora, Nuevo León y Querétaro, siendo este último el que presenta mayor inversión extranjera.

\section{Industria aeroespacial en Chihuahua}

Chihuahua cuenta con un amplio desarrollo tecnológico lo que le permite incursionar en grandes mercados y se ha planteado objetivos para sobresalir también en la industria aeroespacial. Cuenta con 29 empresas de manufactura, 37 proveedores certificados bajo los estándares internacionales de la industria y cinco ensambladoras de equipos originales, por lo que se ubica en el tercer lugar a nivel nacional. Todas estas empresas, agrupan una inversión de más de 1.5 billones de dólares en el sector y generan más de 15 mil empleos directos. Chihuahua dispone del 25\% de las plantas en el país y el 35\% del empleo nacional (Excélsior, 2016). Para el 2021, Chihuahua buscará reducir su dependencia en las importaciones de moldes, herramentales y servicios especializados en un $50 \%$ del actual. 


\section{Industria aeroespacial en Sonora}

La industria aeroespacial sonorense se ha enfocado en la innovación y el suministro de materiales, principalmente en la fabricación de turbinas, por lo que se tienen establecidos objetivos a corto y mediano plazo para ser líder mundial en la fabricación de turbinas, contando con 53 empresas y exportando cerca de 164 millones de dólares, sus principales mercados Estados Unidos, Canadá, Alemania y Francia. Entre las empresas que destacan en Sonora se encuentran: Goodrich, Rolls-Royce, Daher, Grupo Latecoere, Radiall, entre otras, la mayoría de origen estadounidense, francés y británico.

\section{Industria aeroespacial en Baja California}

Baja California, es un estado que se caracteriza por tener una amplia variedad de industrias, desde la alimenticia hasta la manufacturera y eléctrica, y que busca ser el principal productor de la industria aeroespacial. El Estado tiene el potencial para desarrollar sistemas de fuselaje y plantas de poder, pero uno de sus objetivos primordiales es apoyar a toda la producción del país para ser líder en América Latina. Baja California cuenta ahora con 98 empresas dedicadas al sector aeroespacial, empleando a 33,313 personas, algunas son Honeywell, Goodrich, e Eaton Aerospace. En septiembre de este año, una empresa estadounidense llamada "Icon Aircraft" dio a conocer su proyecto donde pretende la instalación de una nueva planta en Tijuana, que comenzará a trabajar en 2017, la cual ofrecerá más de 1,000 empleos y traerá una inversión extranjera de 150 millones de dólares (El Financiero, 2016).

\section{Industria aeroespacial en Nuevo León}

Por su parte Nuevo León es considerada como la entidad del desarrollo tecnológico, sus sectores estratégicos son: aeroespacial, automotriz, biotecnología, electrodomésticos, agroalimentario, servicios médicos especializados, software, nanotecnología, vivienda sustentable y medios interactivos. Además tiene 126 parques industriales y tecnológicos, y acoge a diversas empresas reconocidas a nivel mundial como: CEMEX, FEMSA, VITRO, Cluster Automotriz, entre otras. Específicamente, en el sector aeroespacial, Nuevo León tiene 24 empresas establecidas y sus exportaciones son superiores a 150 millones de dólares anualmente, estas plantas exportan el 90\% de su producción y algunas son: MD Helicopters, Mexcoaero, Monterrey Jet Center, Tecmaq, Metrolab, Frisa Aerospace, Asesa, Aztek 


\section{LA INDUSTRIA AEROESPACIAL: UNA ANÁLISIS DE SU PRODUCTIVIDAD EN MÉXICO}

Technologies, Estampados Monterrey, Ezi Metales, Exova, Hemaq, Herramental Monterrey, Jaiter, Metalinspec y Viakon.

\section{Industria aeroespacial en Querétaro}

En términos aeroindustriales, Querétaro se especializa en el diseño de turbinas, manufactura, ensamblado y mantenimiento, reparación y uveral de partes complejas de fuselajes, turbinas y trenes de aterrizaje. Adicionalmente, el gobierno del estado proporciona apoyos para el crecimiento de la industria aspirando a ser líder mundial, debido a grandes oportunidades que ofrece.

Querétaro, tiene gran apertura comercial, por lo que recibe inversión extranjera directa y gracia a ello muchas empresas se han establecido ahí, también su ubicación es estratégica, se posiciona en el segundo lugar en ciencia y tecnología, su infraestructura es de calidad y ocupa el cuarto lugar en productividad científica e innovadora.

El Estado está conformado por 25 empresas de manufactura y proveedores, tres empresas de mantenimiento y reparación, cinco centros de diseño e ingeniería, tres centros de innovación y desarrollo, y tres instituciones educativas.

Entre las empresas asentadas en Querétaro, con mayor representatividad en el sector, se encuentran:

- Industria General Electric, enfocada a la manufactura y reparación de motores grandes.

- Snecma/Safran, orientada a la producción de motores medianos y su reparación.

- Industria de Turbo Propulsores, dedicada a la fabricación de turbinas de baja presión y a su mantenimiento.

- Bombardier, es una empresa de origen canadiense de las más importantes y que se ha establecido en Querétaro gracias a las oportunidades que ofrece, aunque anteriormente solo se dedicaba a la producción de ferrocarriles, desde 1986 incursionó en este sector, está consolidado como el cuarto mayor fabricante aeronáutico del mundo, y la planta que creó en Querétaro se encarga de la producción de los componentes de los aviones Lear jet 85, ubicándose dentro del parque aeroespacial de Querétaro, y sus operaciones comenzaron en 2013.Bombardier ofrece alrededor de 240 empleos, exportó los primeros cinco prototipos de fuselajes del avión Learjet 85, y en Querétaro se produce 85\% de este avión de última generación. 


\section{LA INDUSTRIA AEROESPACIAL: UNA ANÁLISIS DE SU PRODUCTIVIDAD EN MÉXICO}

\section{Creación de Aerocluster}

Con la productividad de las empresas mexicanas se ha creado una organización sin fines de lucro que reúne a las empresas del sector aeroespacial para su integración en el mercado que trabaja en la incorporación de los proveedores locales a la cadena de valor nacional e internacional, "Aerocluster".

Chihuahua aún no cuenta con este tipo de organización, pero está el Clúster Aeroespacial de Chihuahua, que de igual manera busca el crecimiento de sus empresas. Sonora, Nuevo León, Querétaro y Baja California si cuentan con "Aerocluster", lo que facilita su introducción en el mercado internacional.

Existen desventajas que ponen en riesgo el desarrollo de esta industria siendo la inseguridad y corrupción las principales, ejemplo de esto es la industria ubicada en Chihuahua que es uno de los estados más inseguros y violentos. Sin embargo, puede ser un mercado estratégico debido a su ubicación y a la frontera con los Estados Unidos, lo que puede facilitar las exportaciones.

Nuevo León es un mercado muy competitivo, ya que tiene experiencia en todo tipo de industrias, es uno de los Estados más industrializados, es por ello que esperan que el sector aeroespacial también les permita ser uno de los mejores a nivel nacional y porque no, internacional. Asimismo, el Estado que está a la mira de todos es Querétaro, ya que en los últimos años es el que cuenta con más oportunidades de crecimiento para pequeñas y medianas empresas, por lo que su inversión extranjera ha aumentado considerablemente.

\section{Análisis cuantitativo de la industria aeroespacial mexicana}

La figura 1 muestra las exportaciones de México, con un crecimiento lento entre 2004 y 2009 siendo años difíciles para la industria. El mayor auge se da entre 2010 y 2013 donde las exportaciones ha aumentado hasta 5463000 de dólares. 


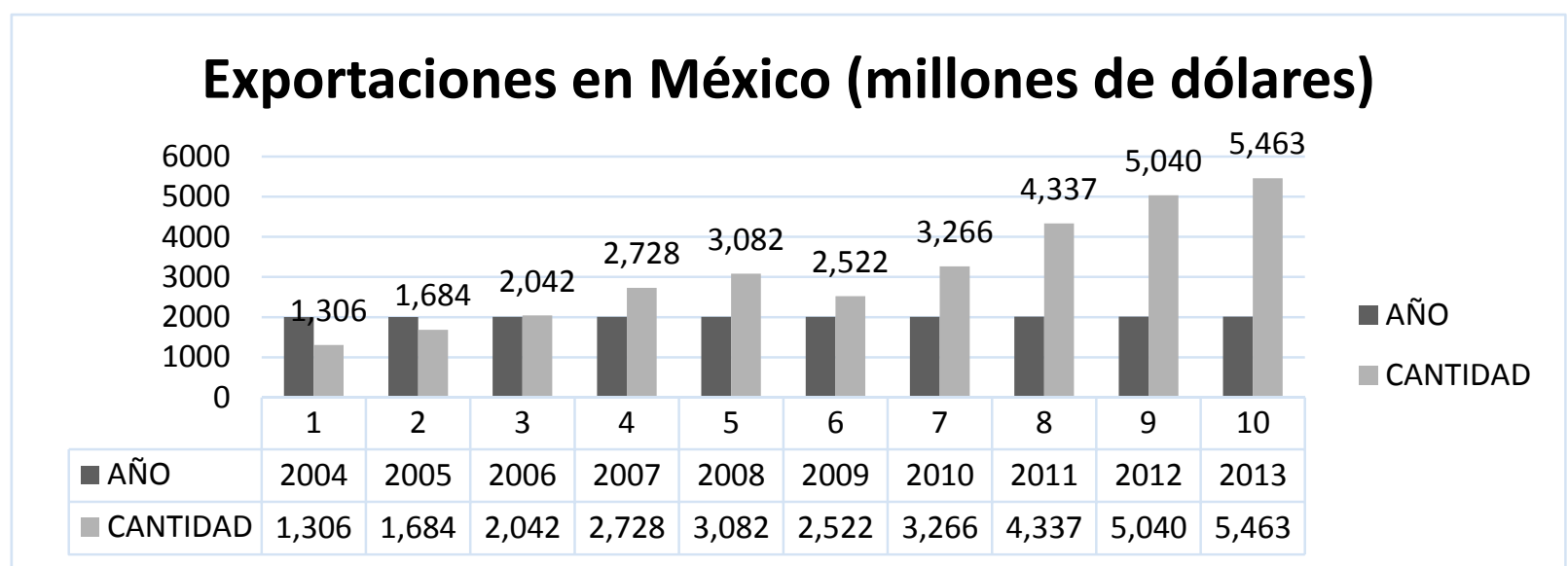

Figura 1. Exportaciones de México de la industria aeroespacial desde el año 2004 al 2013. Fuente: Secretaría de Economía, 2015.

La tabla 1, muestra las empresas que proveen de partes de aeronaves al país, así como los modelos de los aviones. Se observa, que es Airbus la principal compañía proveedora en México seguida por Embraer.

Tabla 1

Proveedores con presencia en México

\begin{tabular}{lcc}
\hline Empresa & Modelo & No. de proveedores \\
\hline Airbus & $330-300$ & 16 \\
Airbus & A380 & 20 \\
Boeing & 787 & 14 \\
Boeing & $747-400$ & 12 \\
Bombardier & CRJ200 & 13 \\
Embraer & ERJ 135 & 17
\end{tabular}

Fuente: FEMIA, 2014.

En la tabla 2, se puede apreciar a las empresas que tienen presencia en Chihuahua y las principales áreas de servicio al que se enfocan. 


\section{LA INDUSTRIA AEROESPACIAL: UNA ANÁLISIS DE SU PRODUCTIVIDAD EN MÉXICO}

Tabla 2

Empresas dedicadas a la producción de partes de aeronaves presentes en Chihuahua

\begin{tabular}{ll}
\hline \multicolumn{1}{c}{ Empresas } & \multicolumn{1}{c}{ Área } \\
\hline Honeywell Aerospace & Fabricación de turbinas \\
Labinal Safran Group & Arnés e ingeniería y diseño. \\
SGI- Electro Switch & Electrónico \\
Textron Cessna & Arnés \\
Aerosan & Estructuras metálicas \\
Cambrian Industries & Maquinado \\
Capsonic Automotive \& Aerospace & Partes para turbina \\
|Hawker Beechcraft Corporation & Helicópteros \\
Zodiac & Productos para emergencia \\
Air Crussiers & Partes de aviones \\
\hline
\end{tabular}

Fuente: Secretaría de Desarrollo Industrial (2013).

Dentro de la tabla 3 se enlistan el total de empresas establecidas en el estado de Sonora del año 2009 al 2015, notándose un incremento de 28 empresas entre dicho período y una duplicación en el número de empleos provenientes de la industria.

\section{Tabla 3}

Empresas establecidas en Sonora

\begin{tabular}{|c|c|c|}
\hline 2009 & 2013 & 2015 \\
\hline 38 empresas & 45 empresas & 60 empresas \\
\hline 5,000 empleos & 7,500 empleos & 10,000 empleos \\
\hline $\begin{array}{l}\text { Capacidades: } \\
\text { Materiales compuestos }\end{array}$ & $\begin{array}{c}\text { Nuevas capacidades: } \\
\text { Aero estructuras }\end{array}$ & $\begin{array}{l}\text { Nuevas capacidades } \\
\text { Turbinas: }\end{array}$ \\
\hline $\begin{array}{l}\text { Turbinas: } \\
\text { • Maquinados simples. }\end{array}$ & $\begin{array}{l}\text { Turbinas: } \\
\text { - Maquinados de precisión. } \\
\text { - Tratamientos térmicos. } \\
\text { - Tratamientos químicos. }\end{array}$ & $\begin{array}{ll}\text { - } & \text { Forjado. } \\
\text { - } & \text { Fundición. } \\
\text { - } & \text { Recubrimientos } \\
& \text { superficiales. }\end{array}$ \\
\hline
\end{tabular}

Fuente: Secretaría de Economía del Estado de Sonora (2015).

Por otra parte, la figura 2 ejemplifica el incremento en la tasa de empleo entre el año 2004 y el 2007 en el estado de Baja California, elevando el número de empleos del año 2006 que se contaban con 1380 a 2279 en el 2007. 
LA INDUSTRIA AEROESPACIAL: UNA ANÁLISIS DE SU PRODUCTIVIDAD EN MÉXICO

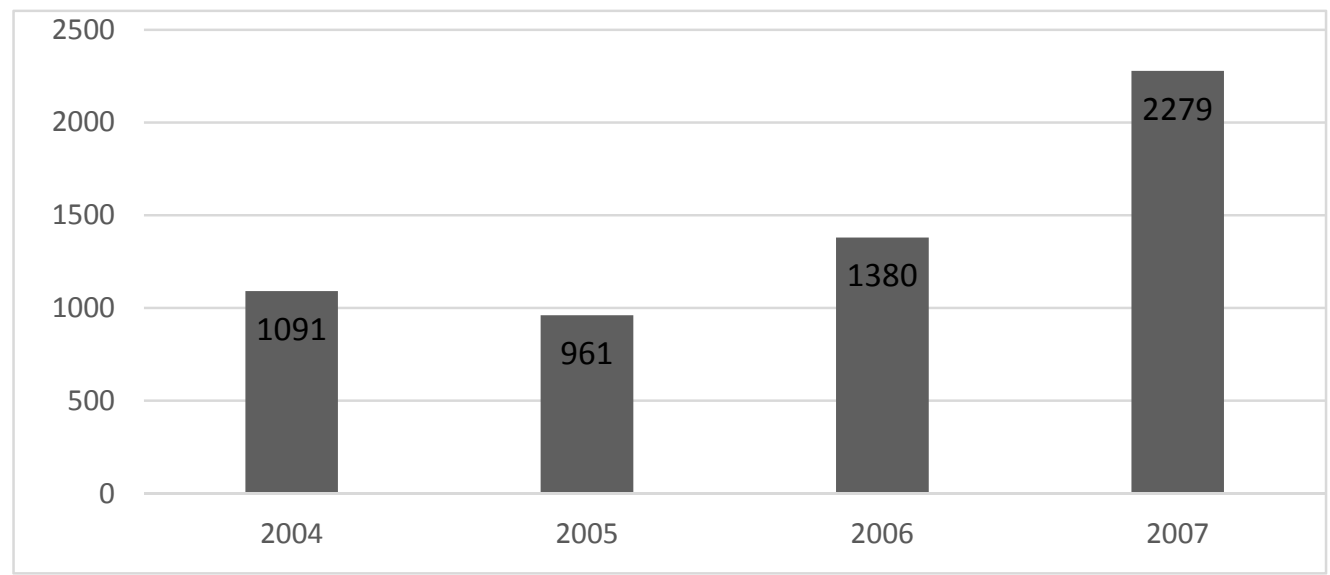

Figura 2. Total de empleos por ciudades de Baja California.

Fuente: Aerospace Industry Directory, 2011.

La tabla 4 describe los clústers distribuidos en cada Estado de la República, así como el giro que tienen y el número de empresas que lo conforman.

\section{Tabla 4}

\section{Principales clústers en México}

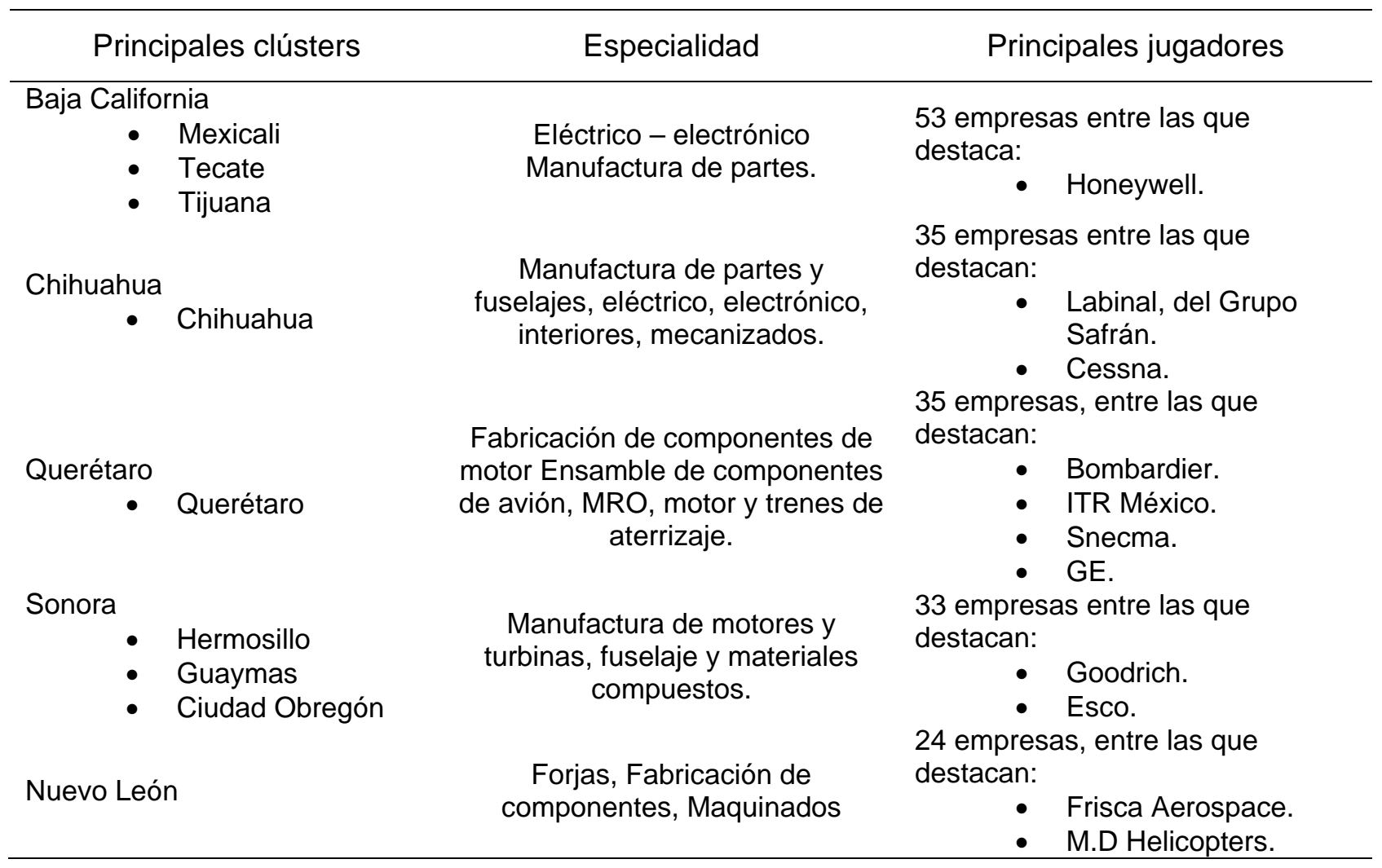

Fuente: FEMIA, 2015. 
LA INDUSTRIA AEROESPACIAL: UNA ANÁLISIS DE SU PRODUCTIVIDAD EN MÉXICO

La figura 3 expone el número de aeronaves que se solicitan a nivel mundial, disminuyendo en el año 2005, pero recuperándose en el 2006 con un total de 1, 380 importaciones.

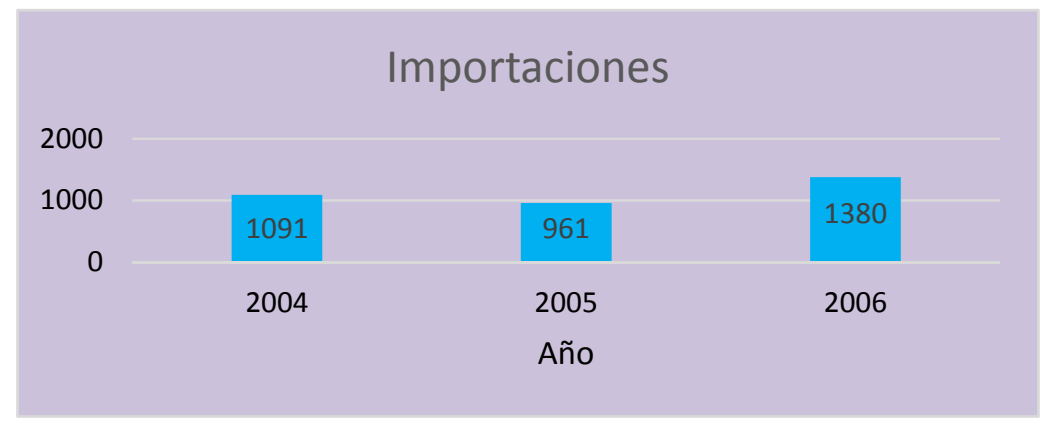

Figura 3. Solicitud de aeronaves a nivel global.

Fuente: FEMIA (2015).

La tabla 5, muestra el total de ingresos en millones de dólares de la industria aeroespacial a nivel mundial, México se encuentra en la posición número 15, con una participación del 0.7\%. El concierto mundial lo encabezan Estados Unidos y Francia.

Tabla 5

Ingresos de la industria aeroespacial por países, 2009 (Millones de dólares)

\begin{tabular}{lcc}
\hline País & Ingresos & Participación (\%) \\
\hline Estados Unidos & 204.0 & 45.3 \\
Francia & 50.4 & 11.2 \\
Reino Unido & 32.7 & 7.3 \\
Alemania & 32.1 & 7.1 \\
Canadá & 22.3 & 5.0 \\
Japón & 14.2 & 3.2 \\
China & 12.0 & 2.7 \\
Rusia & 10.0 & 2.2 \\
Italia & 9.9 & 2.2 \\
Brasil & 7.6 & 1.7 \\
España & 6.1 & 1.4 \\
Singapur & 4.3 & 1.0 \\
India & 4.0 & 0.9 \\
Países Bajos & 3.4 & 0.8 \\
México & 3.0 & 0.7 \\
Otros & 34.2 & 7.6 \\
Total & 450.0 & 100.0 \\
\hline
\end{tabular}

Fuente: Banco Mundial (2009). 


\section{CONCLUSIONES}

México está destacando en la industria aeroespacial, reflejada a través del número de exportaciones que está realizando actualmente, debido al interés que le está dando los gobiernos locales y federales, el rezago que tenía hace más de 40 años empieza a mermar teniendo un auge tecnológico y tomando en cuenta zonas estratégicas del país que realmente están contribuyendo a que sea posible.

Cabe destacar, la participación de los diferentes organismos que se han creado para contribuir a esta industria. Sin embargo, se debe trabajar en zonas que requieren un crecimiento para mejorar su economía, y mantener una estabilidad del país.

México es líder mundial en el sector aeroespacial con un crecimiento del $17.2 \%$ en promedio y el sexto lugar entre los países que más exportan a la industria aeronáutica de Estados Unidos, en 2014 las exportaciones del sector aeroespacial mexicano fueron de 6 mil 366 millones de dólares, en 2015 las exportaciones fueron de 7 mil 500 millones dólares.

Actualmente, la industria Manufacturera aeroespacial cuenta con mano de obra técnica y profesional a nivel nacional que puede competir con el capital humano de cualquier país del mismo sector y rama competitiva.

\section{REFERENCIAS BIBLIOGRÁFICAS}

Aeroclúster Querétaro (2016). Facilitación y vinculación con agencias y empresas de certificación Aeroespacial $y$ de calidad. Recuperado de http://www.aeroclusterqueretaro.mx/

Aerospace Industry Directory (2011). Directorio de la Industria Aeroespacial. Recuperado de http://www.investinbaja.gob.mx/docs/en/industries/aeroespacial/Directorio_Aerospace_BC. pdf

De la Madrid, E. (2016). La industria aeroespacial y el despegue de la productividad en México. Comercio Exterior. Recuperado de http://www.revistacomercioexterior.com/articulo.php?id=54\&t=la-industria-aeroespacial-yel-despegue-de-la-productividad-en-mexico 
LA INDUSTRIA AEROESPACIAL: UNA ANÁLISIS DE SU PRODUCTIVIDAD EN MÉXICO

Becerra, A. (2015). La integración de la economía de Sonora a la industria aeroespacial. Universidad de Sonora (Tesis de maestría). Universidad de Sonora, México.

Casalet, M. (2013). La industria aeroespacial: complejidad productiva e institucional. México: FLACSO.

Consultores internacionales. (2011). Sector secundario en México. Recuperado de http://www.consultoresinternacionales.com/publicaciones/ciscomentario/510/CISComentari oNo510.pdf

Lara, J. (2014). Lidera NL en el sector aeroespacial. El financiero. Recuperado de http://www.elfinanciero.com.mx/monterrey/lidera-nl-en-el-sector-aeroespacial.html

Logística Énfasis (2016). Destaca Chihuahua en la industria aeroespacial. Recuperado de http://www.logisticamx.enfasis.com/notas/74546-destaca-chihuahua-la-industriaaeroespacial

Estrella, V. (2016). Industria aeroespacial requiere investigadores y técnicos: KS. El Economista. Recuperado de http://eleconomista.com.mx/estados/queretaro/2016/11/01/industria-aeroespacial-requiereinvestigadores-tecnicos-ks

FEMIA. (2015). Pro-Aéreo 2012 - 2020 Programa Estratégico de la Industria Aeroespacial. Recuperado de http://economia.gob.mx/files/comunidad_negocios/industria_comercio/PROAEREO-12-032012.pdf

Flores, J. (2011). México piensa positivo. México: Felou.

HSBC. (2016). Principales importaciones de México. Recuperado de https://globalconnections.hsbc.com/mexico/es/articles/principales-importaciones-de-mexico Hualde, A. \& Carrillo, J. (2006). La industria aeroespacial en Baja California. Características productivas y requerimientos actuales y potenciales de capital humano. Pp. $19-21$.

INEGI. (2015). Economía de México. Recuperado de http://cuentame.inegi.org.mx/economia/default.aspx?tema=E

INEGI. (2016). Estadísticas del Comercio Exterior de México. Recuperado de http://www.inegi.org.mx/est/contenidos/espanol/proyectos/metadatos/continuas/ece_334.as $p ? c=10999$ 


\section{LA INDUSTRIA AEROESPACIAL: UNA ANÁLISIS DE SU PRODUCTIVIDAD EN MÉXICO}

Medina, S. (2012). El despegue de la industria aeroespacial en México. Bancomext. Recuperado de http://revistas.bancomext.gob.mx/rce/magazines/151/1/Nov-Dic_3-9.pdf

Mundo Ejecutivo. (2016). Industria aeroespacial por alcanzar los 8 mil mdd. Recuperado de http://mundoejecutivo.com.mx/videos/2016/10/12/industria-aeroespacial-por-alcanzar-8-milmdd

Negocios. (2016). México busca entrar al top ten en la industria aeroespacial. Recuperado de http://mundoejecutivo.com.mx/economia-negocios/2016/03/31/mexico-busca-entrar-topten-industria-aeroespacial

OCAV. (2015). Diagnóstico del sector en México y a nivel internacional. Recuperado de https://www.gob.mx/cms/uploads/attachment/file/58801/Diagn_stico_del_sector_en_M_xico _y_a_nivel_internacional.pdf

Oxford Business Group. (2015). El sector industrial de México: listo para el crecimiento. Recuperado de http://www.oxfordbusinessgroup.com/news/el-sector-industrial-dem\%C3\%A9xico-listo-para-el-crecimiento

PROMÉXICO. (2016). Anuncian inversiones en la industria aeroespacial de México. Recuperado de https://www.gob.mx/promexico/articulos/anuncian-inversiones-en-laindustria-aeroespacial-de-mexico

Secretaría de Economía. (2015). Aeronáutico. Recuperado de http://www.economiasonora.gob.mx/portal/aeronautico-aeroespacial

Tecnológico de Monterrey. (2011). Exportaciones de México. Recuperado de http://www.ciltec.com.mx/es/comercio-exterior/exportaciones-de-mexico

Tovar, E. (2016). Industria aeroespacial mexicana: Panorama 2016. Modern Machine Shop. Recuperado de https://www.mms-mexico.com/art\%C3\%ADculos/industria-aeroespacialmexicana-panorama-2016

Visión industrial. (2012). Universidad Nacional Aeronáutica en Querétaro. Visión industrial. 